\title{
Uncertainty management of situations in a housing use context
}

\author{
Cedric DEFFO SIKOUNMO \\ Univ. Savoie Mont Blanc \\ LISTIC, F-74000 Annecy, France \\ Email: cedric.deffo-sikounmo@univ-smb.fr
}

\author{
Stephane PERRIN \\ Univ. Savoie Mont Blanc \\ LISTIC, F-74000 Annecy, France \\ Email: stephane.perrin@univ-smb.fr
}

\author{
Eric BENOIT \\ Univ. Savoie Mont Blanc \\ LISTIC, F-74000 Annecy, France \\ Email: eric.benoit@univ-smb.fr
}

\begin{abstract}
This paper proposes a new contribution to manage uncertainty in the context of a situation recognition. It gives an application to the measurement of housing use situations. It extends a previous communication that proposes an ontological based model of situations. In this model, a situation is a set of entities linked by relationships themselves determined using sensors. However, such model doesn't allow to manage uncertainty. In order to take into account an uncertainty related to the identification of some entities, we propose to model the set of possible eligible entities, called the universe of discourse, by an anonymous instance in the ontology. This anonymous instance is linked with a "same as" relationships with all possible eligible entities. In addition, we propose to complete this model by the association of a mass with each "same as" relationship. This paper presents a probabilistic semantic to these masses. A simple didactic example on a housing use situations measurement illustrates the presented approach.
\end{abstract}

\section{INTRODUCTION}

The Iot field sees the raise of sensible objects that are able to perceive their close environment. By the way, in a community of sensible objects, any object might be able to perceive the other close objects or more generally any physical or biological entity close to it. As a projection of the global state of the entity community on a subset of entities, a situation gives a higher vision of a given environment. In this paper, we focus on the recognition of situations in a housing use context. In this case the biological entities are peoples, children or pets and the physical objects are furniture units and household appliances. The sensible objects are then intelligent connected furniture units [1] and connected appliances. The goal of the situation recognition in a housing use context is to identify the human uses of the house in order to improve their day-to-day experience. The application fields of the presented study are the assistance to people not only for comfort but also for home support of elderly persons and disabled persons. Indeed, the dynamic identification of human uses allows to detect changes in daily routines as it appends when an underlying medical or health issue appears.

In a previous study, we presented the advantage of representing the global state of the entity community with a factual ontology [2]. With this approach, a situation is considered to be realized when the ontology that defines this situation is aligned with the factual ontology. In other words, it is realized if one or more instances of this situation definition exists in the factual ontology that describes the global state.
This approach supposes that the facts that define the global state are perfectly known. But in a context of a community of objects including sensible objects, some facts are deduced from objects perceptions and are rarely perfectly known. Indeed, as a fact is deduced from measurement results given by low cost sensors. In this case the uncertainty introduced by the measurement is significant. The identification of the situation is provided by a fusion process that uses these measurement results as inputs. Therefore the management of uncertainty is a critical point in the situation identification process.

The final goal of the study presented in this paper is to produce an information on the uncertainty on the realization of a situation. A first chapter will remind the concept of ontologies and its application to the definition of situations then its application to the identification of the realized situations. A second chapter will present the limits of the usual approaches used to consider uncertainty in the factual ontologies. The third chapter is devoted to the presentation of our approach including the method to represent a universe of discourse on the factual ontology and the method to model the uncertainty according several theories.

\section{Situation in A Housing USE CONTEXT}

Let us define an environment made of humans and of household objects including smart connected furniture units. Let us note the various entities describing or influencing the different states of our environment. As an example, a living room can be chosen as a context for the environment. In the same example, the room can be made up of a sofa, chairs, a coffee table, a TV cabinet, a television and living occupants. We remind that the intelligent connected furniture units are sensible objects that hold embedded sensors: the sofa and the chairs may have sensors that allow them to detect if a human sit on them or that allow to perceive the proximity of other objects or of humans. It is also assumed that the sofa dialog with the coffee table itself in communication with the television, which allows an occupant to manage the TV from the sofa. Let admit that the following states can be detected: "occupants watch a movie", "an occupant sits on the couch", "an occupant is watching TV", "occupants are discussing". From the above, we can describe our environment as a community of actors of various natures who interact. We first propose to split the actor category into the human category 
and the object category. The object category generalizes the smart connected object category that generalizes the smart connected furniture unit category. To find the best way to represent the knowledge of our environment, we need a representation that must be structured, portable, scalable and dynamic while keeping the semantics that corresponds to the environment. To perform this representation we choose the ontologies that already apply in different fields of research namely: knowledge engineering, automatic natural language processing, cooperative information systems and many others.

\section{A. Ontology}

The notion of ontology associated with the artificial intelligence appeared early 1990s. The most cited definition is the Gruber's one [3], he defines an ontology as "an explicit specification of a conceptualization". A conceptualization is a common vision of an object of the real world that can be physical or abstract. In 1997, the definition given by [4] is more refined and specifies that the conceptualization must be shared. Conceptualization should express a shared vision of the described object. Therefore that can easily be interpreted by software. The years after present an ontology as the result of a modeling by emphasizing the characterization of primitives for the representation of knowledge. Finally, in [5], Bouquet et al give the following definition: "Ontologies are shared models of a domain that encode a view which is common to a set of different parties". The various and complementary views of these definitions can be noted. Thus, an ontology defines a common and shared vocabulary for a particular domain. This allows communication between agents. The ontologies are designed to facilitate the exchange and reuse of vocabularies.

Hence, the parts of an ontology are:

- classes modeling the entities of the real world. These classes named concepts can be organized hierarchically by a subsomption relation named inheritance. Human, furniture unit or TV are examples of classes.

- Properties expressing relations and interactions between classes or describing class attributes.

- Axioms formalizing verified interactions of the real world. Example : $($ Woman $=$ Person $)$ and $($ Sex $=$ Female) .

- Individuals representing the concrete concept instances. Example: (Paul is_instance_of Person).

In addition, an ontology must satisfy the principles of clarity, objectivity, completeness, consistency, extensibility and modularity as described in [6]. A language must be adopted to describe the ontologies in a homogeneous way. The most common one are currently RDFS and OWL (Web Ontology Language) [7]. In [8], the authors briefly describe the evolution of these descriptive languages until the completion of the OWL languages family.

\section{B. Situation recognition}

In this study, a situation is defined as a state of a given set of physical or biological entities called "actors of the situation". A situation has an abstract view that we call the reference situation. The situation recognition is then the identification of a realization of the reference situation in the real world.

1) Reference situation: In [2], a reference situation is then defined by the actor types (called concepts), and by a set of relations between these concepts.

2) Realized situation: A realized situation is the instance of a reference situation. It's a state involving a limited set of actors. In other words, it's a state involving the actors defined in the reference situation. A situation is identified as realized if the factual ontology includes the instances of the concepts and of the relationships that define the reference situation.

Thus, the truth level of the realization of a situation directly depends on the truth level of the relationships defined in the reference situation. As the thruth level of the relationships is given by the measurement performed by the sensible objects, it may hold an uncertainty that must be managed in the ontology.

\section{A SHORT RESUME OF UNCERTAINTY MANAGEMENT ON ONTOLOGIES}

\section{A. Uncertainty management}

Given a smart connected furniture unit embedding sensors of different natures, these sensors send various information on quantities like temperature, pressure, vibration, location. However, the reliability of this information is not always assured. The imperfections of the information can be:

- ambiguity when the returned information doesn't enable to distinguish two different states,

- noise that holds a non-pertinent part of the information,

- bias that reflects the unknown of potential information sources,

- incompleteness when some information is missing,

- inaccuracy,

- uncertainty,

- inconsistency,

- redundancy.

In order to consider these imperfections, at least the uncertainty must be managed on the representation introduced section II-A.

\section{B. Uncertainty management on ontologies}

Regarding the advance of domains such as data acquisition and information fusion, it was necessary to find ways to associate the logical uncertainty management formalisms with the knowledge representation methods. Indeed, there are several formalisms to manage incomplete information as well as information related uncertainty. Some studies aimed at extending the OWL language and the description logic to the mathematical formalisms supporting uncertainty. This is reflected in the advent of languages such as: PR-OWL, FuzzyOWL, BayesOWL. See for example [9] for a survey on the methods and languages. In the literature, the probability theory is the most used for the uncertainty management. In 2006, Da Costa [10] proposed a first version for PR-OWL: a probabilistic generalization of the OWL language. In PR-OWL the semantics is based on Multi- Entity Bayesian Networks (MEBN) [11]. The MEBN are made of fragments denoted 
Mfrags that represent the probable information related to a set of random variables. However, this version specifically focuses on the MEBN semantics. Hence the proposal of PROWL2 in [12] which places more emphasis on expressiveness and on OWL semantics. The PR-OWL approach is to create a meta-ontology where uncertain concepts are defined with superclasses. In the same context, we can find BayesOWL [13]. This kind of ontology, based on Bayesian networks, gives a solution to combine knowledge with observations and a statistic learning theory. BayesOWL gives a set of rules and processes to simplify the migration from OWL to RBs while maintaining the semantics expressed on the initial ontology. Finally, FuzzyOWL presented in [14] are ontologies based on the fuzzy subset theory with an extension to the description logic named fuzzyDL. In this case, each concept of the ontology is associated to a "fuzzy concept". Properties and axioms are also respectively associated to their respective fuzzy versions.

\section{Why a new proposal?}

The advantages of these ontology languages lies in their ability to reason. Indeed, the theories of mathematical logics on which they are based have proved their advance in the field of modeling, representation and uncertainty management. These studies try to make classes and concepts uncertain but in our approach the concept ontology is stable and only its instances, the factual ontologies, are subject to the uncertainty. Indeed, in our approach the facts are permanently moving and the factual ontology reflects our knowledge on these facts including the uncertainty on the facts truth.

\section{A NEW PROPOSAL}

In the field of description logics, knowledge modelling of a domain is performed on two levels. The first level corresponds to the TBox statement that describes the domain general knowledge. The second level corresponds to the ABox statement, also called factual level, that represents a fact, a kind of "instance" related of one configuration. A TBox component includes definition of concepts and roles, and an ABox component describes each individuals by naming them and specifying assertions on individuals that are named using concepts and roles. Several ABox can be associated with a same TBox; each ABox represents a configuration based on a set of individuals and uses concepts and roles included in the TBox. In this paper, we propose to introduce uncertainty. In our case, the uncertainty comes from the measurements. Measures are instanced and therefore are modeled inside ABox. This section describes our proposal to model uncertainty into ABox.

\section{A. Problem definition}

As shown in section 1, a furniture environment is described by a factual ontology. This ontology describes both static knowledge and dynamic one. Our goal is to retrieve realized situations of our environment and to associate a truth level to each realized situation. According to this goal, the uncertainty

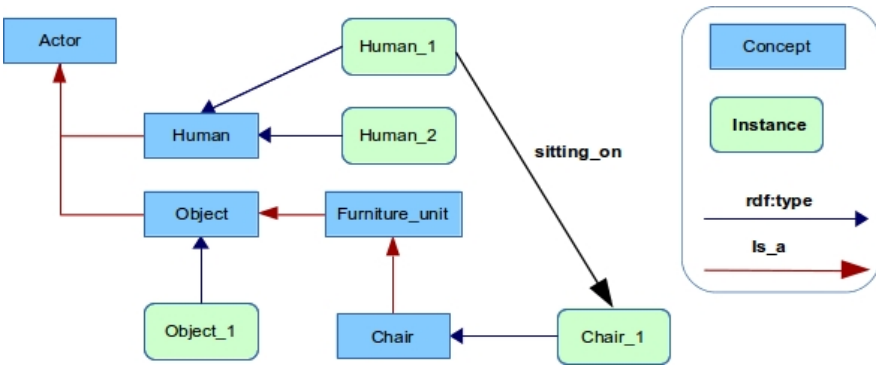

Fig. 1. This figure presents a concept ontology (named Tbox), a factual ontology (named Abox or instance ontology) and the $r d f$ : type relation that links them. In this factual ontology, we can recognize a situation where a human is sitting on a chair.

management has to be applied on this ontology based representation. Before defining any meaning for this truth level we will simply call it mass. Depending on the theory chosen to model the uncertainty, the mass will represent for example a probability, a possibility or a belief mass.

A situation corresponds to a graph where relations corresponds to services given by nodes. Nodes represent a piece of furniture or other objects that constitute our environment. For example, suppose that the presence of two humans and a chair is detected in a room. Sensors equip chair allowing it to deliver its state: human is on itself, or is not. Fig. 1 illustrates the ontology of such environment. Suppose that a chair detects that a human is sit on it. On one hand, we want to know which human is sitting on it. More precisely, we want to know the mass associated to the situation "a human is sitting on" for each human candidate. On the other hand, the situation "a human is sitting on" can be uncertainty too. This uncertainty is also represented by another mass. In our precedent work [2], we proposed a model for the representation of situations. But this model does not take into account uncertainty.

\section{B. Representing a universe of discourse in a factual ontology}

The preliminary action to take uncertainty into account is to define the so called universe of discourse that specifies the set of eligible candidates. Fig. 1 illustrates that Human_1 is sitting on chair_1 and represents a certain knowledge. In the real case, the only certain knowledge is that Human_1 and Human_2 are possibly sitting on Chair_1. We need to express in the one hand that a human is sitting on Chair_1. In the other hand that Human_1 and Human_2 are candidates. To express the first need, we propose to use the sitting_on relation to link an anonymous instance with Chair $_{1}$ as illustrated in fig. 2. At this step, no individual fits to the anonymous instance. We just modeled on the factual ontology the fact "something is sitting on Chair_1". To express the set of candidate individuals, we propose to use the owl:sameAs relation to link the anonymous instance to all candidate individuals as shown in fig. 3. The universe of discourse we propose is then made of all owl : sameAs relationships related to the same anonymous instance. As such knowledge is produced by sensor measurements, the universe 
of discourse is expressed on the factual ontology (i.e. the ABox).

This universe of discourse can be formalized using a RDF format triplets:

$<$ Inst $_{i}$ rdf : type Concept $^{i}$, Relation $_{k}$, Inst $_{j}$ rdf : type

Concept $^{j}>$

where $r d f$ : type is the RDF relation that links an instance to its concept.

This triplet expresses that:

- Inst $_{i}$ is an instance of Concept ${ }^{i}$,

- Inst $_{i}$ is linked to Inst $_{j}$ throw the relationship Relation $_{k}$,

- Inst $_{j}$ is an instance of Concept ${ }^{j}$.

An anonymous instance without type, as shown in fig. 3 is represented by (_ :?).

An anonymous instance with a type, as shown in fig. 4 is represented by (_ :? rdf : type Concept $\left.{ }^{i}\right)$.

Let's considering example introduced in section I and the corresponding ontology is presented in fig. 1 to illustrate the of universe of discourse usage. When Chair_1 indicates that sitting_on is an active relationships, the ontology illustrated in fig. 1 becomes the ontology illustrated in fig. 2 . Note that an anonymous instance appeared and is linked to the instance Chair_1 using the sitting_on relationship. From this step, the anonymous instance has no type.

The universe of discourse corresponding to the expression of this knowledge is limited to a single RDF triplet and its negation that expresses "no Human is sitting on Chair_1":

$$
\begin{aligned}
\Omega_{1} & =\{ \\
& <_{-}: ?, \text { sitting_on,Chair_1rdf }: \text { typeChair }>, \\
& \left.<_{-}: ?, \text { sitting_on,Chair_1rdf }: \text { type Chair }>\right\}
\end{aligned}
$$

However, we consider that any instance of ontology is candidate to be the real instance corresponding to the anonymous instance. In our example, Human_1, Human_2, Object_1 and Chair_1 are candidates. As the sitting_on relation is not reflexive, Chair_1 is dropped from the candidates list. The Fig. 3 illustrates this.

The corresponding universe of discourse is:

$$
\begin{aligned}
& \Omega_{2}=\{ \\
& <_{-}: ?, \text { owl }: \text { sameAs, Human_1 rdf : type Human }>, \\
& <_{-}: ?, \text { owl }: \text { sameAs, Human_2 rdf : type Human }>, \\
& \left.<_{-}: ?, \text { owl : sameAs, Object_1 rdf : type Object }>\right\}
\end{aligned}
$$

To illustrate another example of universe of discourse usage, we consider the same previous example except that the type of the anonymous instance is known: anonymous instance is a Human type. In this case, any instance of ontology restricted to the anonymous instance type is candidate to be the real instance corresponding to the anonymous instance. In this example, Human_1 and Human_2 are candidate. Fig 4 illustrates this. The corresponding universe of discourse is:

$$
\begin{aligned}
& \Omega_{2 \text { Human }}=\{ \\
& <_{-}: ?, \text { owl : sameAs, Human_1 rdf : type Human }>, \\
& \left.<_{-}: ?, \text { owl : sameAs, Human_2 rdf : type Human }>\right\}
\end{aligned}
$$

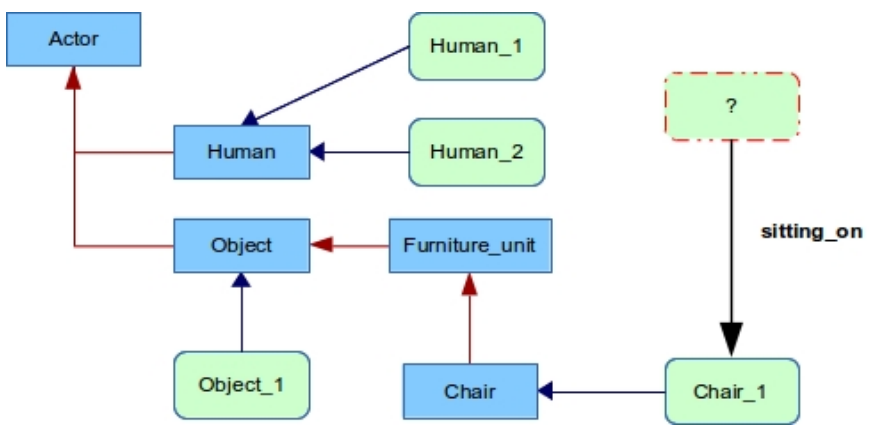

Fig. 2. An anonymous instance is used in the factual ontology to represent the fact that something is sitting on the chair chair_1.

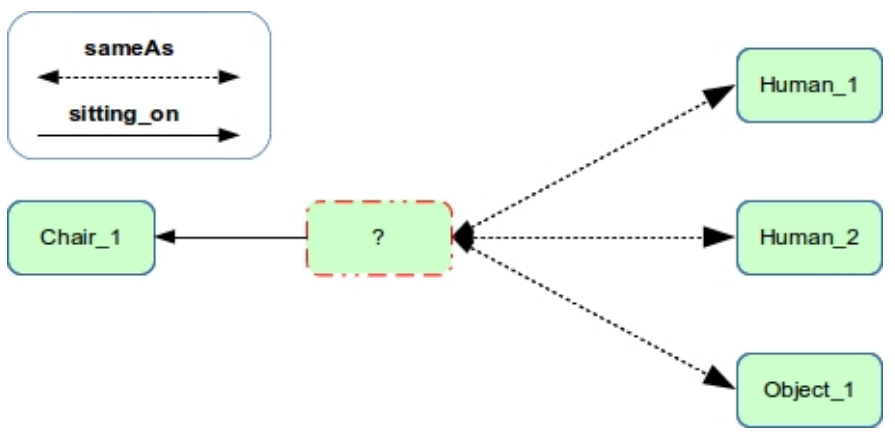

Fig. 3. We propose to use the sameAs relationship to define the set of all instances that possibly be the anonymous instance. This example modelizes the fact that either Human_1, Human_2 or Object_1 are possibly sitting on Chair_1

\section{The uncertainty model}

We propose to use mass to express uncertainty on a knowledge. The wanted result is a set of candidate entities associated with mass for each of them. Each candidate entity can be the real instance corresponding to the anonymous instance. This link is associated with a given mass. In order to model uncertainty into ontology representation, we propose to associate a mass to the owl : sameAs relationship. According to the sources of information, the masses are distributed or rearranged on the related universe of discourse.

Fig 5 illustrates this example. 


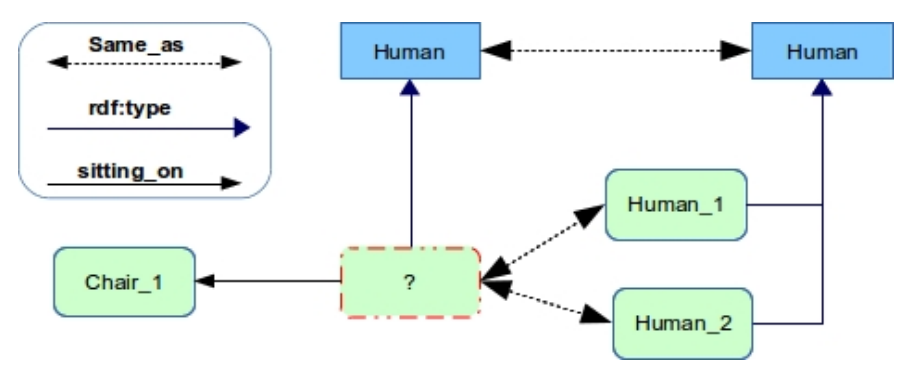

Fig. 4. Known relationships on the anonymous instance act as constraints that must be respected when the alignment is performed. In our example, the ABox expresses the fact that the actor sitting on Chair_1 is a Human and Object_1 is not of Human type so it is no more a possible candidate for the identification of the anonymous instance.

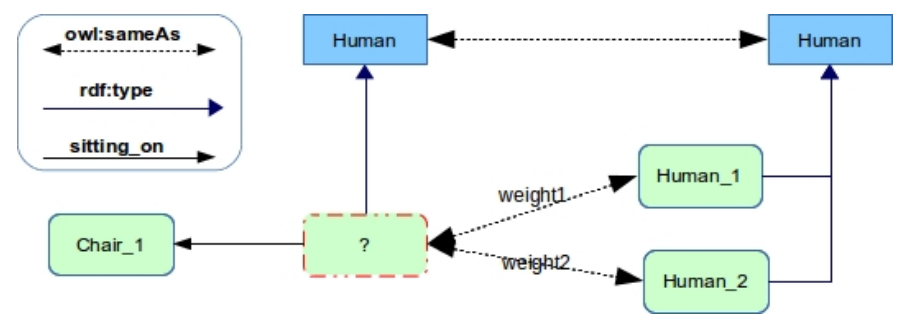

Fig. 5. The owl : sameAs relationships related to a same anonymous node hold the mass that will represent the uncertainty on the indetification of the human that is sitting on the chair.

\section{Managing uncertainty on a factual ontology}

Modeling uncertainty using mass does not include semantic. So, in the first subsection below, we present a probabilistic approach to manipulate the masses; this is traditional approach Then, in the second subsection, we present a possibilistic approach. To facilitate understanding for the reader, we use the same previous example illustrated in fig 5 .

From this examples, the following events can be deduced:

- $E 1=$ "a Human is sitting on Chair_1" ;

- $E 2=$ "Human_1 is sitting on Chair_1" ;

- $E 3=$ "Human_2 is sitting on Chair_1".

At this step of the paper, we already presented the most inovative part, i.e. the knowledge split and the inclusion of the corresponding universes of discourse on the factual ontology. The refinement of the uncertainty management is now quite simple and is detailed below.

1) Probabilistic representation of uncertainty: In the probabilistic modeling, the mass represents the probability of an event. If all possible exclusive events are represented, the sum of each event probability is equal to 1 . In the other case, this sum is less than 1 .

According that $P(E)$ is the probability that an event $E$ occurred, and with respect of the RDF format, $P(E 1), P(E 2)$ and $P(E 3)$ are defined as follow.

$$
\begin{aligned}
& P(E 1)=P\left(<\_: ?, \text { sittingOn, Chair_1 }>\right) \\
& P(E 2)=P(<\text { Human_1, sittingOn,Chair_1 }>) \\
& P(E 3)=P(<\text { Human_2, sittingOn,Chair_1 }>)
\end{aligned}
$$

\section{According that}

$P(\overline{E 1})=1-P(E 1)$ is the probability of the event "no Human is sitting on Chair_1".

Whe have:

$$
\begin{gathered}
P(E 2)=P(<\text { Human_1, sittingOn,Chair_1 }>) \\
=P\left(<<_{-}: ?, \text { sittingOn,Chair_1 }>\right. \\
\cap<\text { Human_1, owl }: \text { sameAs,_:? }>) \\
=P\left(<\_: ?, \text { sittingOn,Chair_1 }>\right) \\
. P(<\text { Human_1, owl }: \text { sameAs,_:? }>)
\end{gathered}
$$

and

$$
\begin{gathered}
P(E 3)=P(<\text { Human_2, sittingOn,Chair_1 }>)= \\
P\left(<\_: ?, \text { sittingOn,Chair_1 }>\right. \\
\cap<\text { Human_2, owl : sameAs, }: ?>)= \\
P\left(<\_: ?, \text { sittingOn, Chair_1 }>\right) \\
. P(<\text { Human_2, owl }: \text { sameAs,_ }: ?>)
\end{gathered}
$$

The most important point to presented here concerns the decomposition of the event "Human_X is sitting on Chair_1" into the 2 events "a human is sitting on Chair_1" and "the human sitting on Chair_1 is Human_X”.

Without external knowledge about Human_1 or Human_2, for example the probability of their presence in the room, the probabilities on the owl : sameAs relationships $P(<$ Human_2, owl : sameAs,_ :? $>)$ and $P(<$ Human_2,owl : sameAs,_:? $>)$ are equals.

In the general case, the ignorance is modeled by a uniform ditribution of the unitary probability over the universe of discourse.

2) Possibilistic representation of uncertainty: In the possibility modeling, masses are representing the possibility that an event occurred. The supremium of the possibilities of exclusive events is equal to 1 . Note that a 0 value for possibility means the impossibility for an event to occur and is already modeled in our approach by no presence of the corresponding relationship. So, for any event $\mathrm{E}$ modeled in the factual ontology, the possibility $\Pi(E)$ respects $0<\Pi(E) \leq 1$.

According that $\Pi(E)$ is the possibility that an event $E$ occurred, and with respect of the RDF format, $\Pi(E 1), \Pi(E 2)$ and $\Pi(E 3)$ are defined from the same example than before.

$$
\begin{aligned}
& \Pi(E 1)=\Pi\left(<\_: ?, \text { sittingOn,Chair_1 }>\right) \\
& \Pi(E 2)=\Pi(<\text { Human_1, sittingOn,Chair_1 }>) \\
& \Pi(E 3)=\Pi(<\text { Human_2, sittingOn,Chair_1 }>)
\end{aligned}
$$


Whe have:

$$
\begin{gathered}
\Pi(E 2)=\Pi(<\text { Human_1, sittingOn,Chair_1 }>) \\
=\Pi\left(<\_: ?, \text { sittingOn,Chair_1 }>\right. \\
\cap<\text { Human_1, owl }: \text { sameAs,_ }: ?>) \\
=\text { min }\left(\Pi\left(<\_: ?, \text { sittingOn, Chair_1 }>\right),\right. \\
\Pi(<\text { Human_1, owl }: \text { sameAs,_:? }>))
\end{gathered}
$$

and

$$
\begin{gathered}
\Pi(E 3)=P(<\text { Human_2, sittingOn,Chair_1 }>) \\
=\Pi\left(<\_: ?, \text { sittingOn,Chair_1 }>\right. \\
\cap<\text { Human_2, owl }: \text { sameAs,_ }: ?>) \\
=\text { min }\left(\Pi\left(<\_: ?, \text { sittingOn, Chair_1 }>\right),\right. \\
\Pi(<\text { Human_2, owl }: \text { sameAs,_:? }>))
\end{gathered}
$$

Without other knowledge about Human_1 or Human_2 i.e. possibility of their presence in the room, the possibility on owl : sameAs relationship $\Pi(<$ Human_2, owl : sameAs,,$\left._{-} ?>\right)$ and $\Pi\left(<H u m a n_{-} 2\right.$, owl : sameAs, ${ }_{-}$: $?>$ ) are equal to 1 .

\section{CONClusion}

The main contribution of this paper is a new proposal to model the universe of discourse on factual ontologies in order to express the uncertainty coming from the measurements performed by sensible objects. The introduction of an anonymous instance to help modeling the universes of discourse is an elegant solution that preserves the original ontology and prevents from the augmentation of its complexity. The proposed approach can be refined to use the usual uncertainty management approaches. The refinements with the probability theory and with the possibility theory are presented in the paper. The application of the evidence theory will be presented later.

The proposed approach helps to model complex interactions between smart connected objects and gives a good support for the identification of situations involving many actors. The significant level of uncertainty produced by the sensible furniture units is now managed in our ontology based model. Indeed the fusion process is able to propagate the given uncertainty in order to identify the situations. Actually the proposed ontological model lays the groundwork of an ambient intelligence that allows us to supervise the evolution of daily routines in order to prevent the consequences of underlying medical or health issues.

Future studies will consist in further expressing various uncertainties, especially when information is provided from several instanced entities, creating a multi-source context. These sources of information can deliver various uncertainty information, that could be contradictory. Evidence theory is an appropriate candidate to manage this kind of information in order to facilitate decision process.

\section{ACKNOWLEDGMENT}

This research is supported by the Universite Savoie Mont Blanc, the Association des Pays de Savoie and Miliboo Corporation.

\section{REFERENCES}

[1] C. D. Sikounmo, E. Benoit, and S. Perrin, "States measurement in a context of intelligent connected furnitures," Journal of Physics: Conference Series, vol. 772, no. 1, p. 012003, 2016.

[2] C. Deffo Sikounmo, E. Benoit, and S. Perrin, Modeling Situations in an Intelligent Connected Furniture Environment. Springer International Publishing AG, 2017, p. to be published.

[3] T. R. Gruber, "A translation approach to portable ontology specifications," KNOWLEDGE ACQUISITION, vol. 5, pp. 199-220, 1993.

[4] W. N. Borst, "Construction of engineering ontologies for knowledge sharing and reuse," Ph.D. dissertation, Enschede, September 1997.

[5] P. Bouquet, F. Giunchiglia, F. van Harmelen, L. Serafini, and H. Stuckenschmidt, $\mathrm{C}$-OWL: Contextualizing Ontologies. Berlin, Heidelberg: Springer Berlin Heidelberg, 2003, pp. 164-179.

[6] A. Gómez-Pérez, "Ontological engineering: A state of the art."

[7] S. Staab and R. Studer, Handbook on Ontologies, 2nd ed. Springer Publishing Company, Incorporated, 2009.

[8] I. Horrocks, P. F. Patel-Schneider, and F. van Harmelen, "From SHIQ and RDF to OWL: The making of a Web ontology language," Web Semantics: Science, Services and Agents on the World Wide Web, vol. 1, no. 1, pp. 7-26, Dec. 2003.

[9] T. Lukasiewicz and U. Straccia, "Managing uncertainty and vagueness in description logics for the semanticweb," Web Semantics: Science, Services and Agents on the World Wide Web, vol. 6, no. 4, 2008.

[10] P. C. G. Da Costa, K. B. Laskey, and K. J. Laskey, "Pr-owl: A bayesian ontology language for the semantic web," in Uncertainty Reasoning for the Semantic Web I. Springer Berlin Heidelberg, 2008, pp. 88-107.

[11] K. B. Laskey, "Mebn: A language for first-order bayesian knowledge bases," Artificial Intelligence, vol. 172, no. 2, pp. 140 - 178, 2008.

[12] R. N. Carvalho, K. B. Laskey, and P. C. G. Costa, "P.c.g.: Pr-owl 2.0 - bridging the gap to owl semantics," in In: International Workshop on Uncertainty Reasoning for the Semantic Web (2010, 2013.

[13] Z. Ding, Y. Peng, and R. Pan, "Bayesowl: Uncertainty modeling in semantic web ontologies," in Soft Computing in Ontologies and Semantic Web. Springer-Verlag, 2006, pp. 3-29.

[14] G. Stoilos, G. B. Stamou, V. Tzouvaras, J. Z. Pan, and I. Horrocks, "Fuzzy OWL: Uncertainty and the semantic web," in OWLED, ser. CEUR Workshop Proceedings, B. C. Grau, I. Horrocks, B. Parsia, and P. F. Patel-Schneider, Eds., vol. 188. CEUR-WS.org, 2005. 